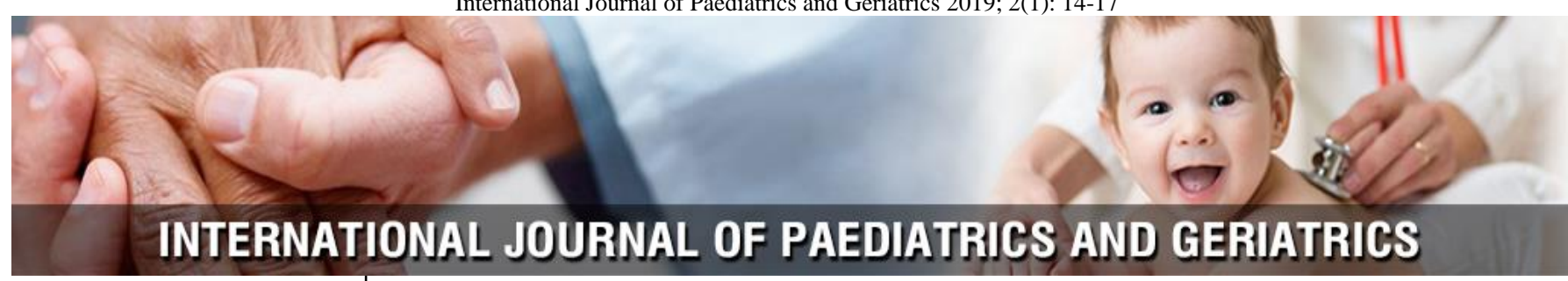

P-ISSN: 2664-3685

E-ISSN: $2664-3693$

www.paediatricjournal.com

IJPG 2019; 2(1): 14-17

Received: 24-11-2018

Accepted: 27-12-2018

\section{Denelle Mohammed}

Saint James School of Medicine 1480 Renaissance Drive, Suite 300 Park Ridge, IL 60068 USA
Corresponding Author: Denelle Mohammed Saint James School of Medicine 1480 Renaissance Drive, Suite 300 Park Ridge, IL 60068 USA

\section{Childhood neglect and autism spectrum disorder}

\author{
Denelle Mohammed
}

DOI: https://doi.org/10.33545/26643685.2019.v2.i1a.22

\begin{abstract}
Childhood neglect is a prevalent issue in today's society and can lead to everlasting psychological, cognitive and behavioral issues. In addition, Autism Spectrum Disorder is becoming more pronounced in pediatric medicine. The objective of this case report is to be a point from which further research can determine if there is a link between child neglect and Autism Spectrum Disorder, so that specific therapeutic interventions can be implemented. The case report describes a 5-year-old male who was exposed to chronic neglect in the early developmental years of his life and was subsequently diagnosed with Reactive Attachment Disorder and Autism Spectrum Disorder, after cognitive and developmental testing. By deducing these specific etiologies and risk factors of Autism Spectrum Disorder, treatment such as behavioral interventions, psychotherapy and cognitive development can be formulated based on evidence-based recommendations in the future. These targeted treatments can assist patients in assimilating into society more successfully.
\end{abstract}

Keywords: Autism spectrum disorder, child neglect

\section{Introduction}

The purpose of this case report is to lead the way to further studies of children who have suffered child neglect and were subsequently diagnosed with Autism Spectrum Disorder, to determine the link between the two. Studies of this nature can paint a more complete picture of the causative and risk factors for Autism Spectrum Disorder, in addition to established genetic studies. Autism Spectrum Disorder as described by the Diagnostic and Statistical Manual-V (DSM-V) includes 1) Autism Spectrum Disorder 2) Pervasive Developmental Disorder not otherwise specified and 3) Asperger's Syndrome. The three main deficits in Autism Spectrum Disorder include abnormal social skills, communication deficits as well as restrictive and/or repetitive types of behavior [1]. Childhood neglect is the most common form of maltreatment ${ }^{[1]}$. According to the U.S. Administration for Children \& Families, Child Maltreatment 2015, approximately $75 \%$ of those who experienced maltreatment or abuse, suffered from childhood neglect. Several of these children go on to suffer from cognitive, social and behavioral issues.

The literature review focused on using academic search engines such as PubMed, EBSCO, Google Scholar, The Lancet, The Journal of the American Medical Association (JAMA) and The New England Journal of Medicine (NEJM) using search terms such as "child neglect and autism link", "risk factors for autism, causative factors of autism", "etiology of autism", "reactive attachment disorder" and "autism spectrum disorder". The literature review provided information on various genetic and neurobiological linkages to Autism Spectrum Disorder. One study stated that decreased cerebellar Purkinje neurons and cerebral cortex dysgenesis has been recognized as the most consistent neurological abnormalities in autistic patients ${ }^{[2]}$. Furthermore, the ENGRAILED 2 gene, implicated in cerebellar development has been associated with Autism Spectrum Disorder ${ }^{[2]}$. Other studies have also been able to replicate the results of that gene trial. Further studies have focused on animal model demyelination causing changes in the prefrontal cortex that have been implicated in Autism Spectrum Disorder ${ }^{[3]}$.

There have also been some environmental risk factors linked to Autism Spectrum Disorder. Autism Spectrum Disorder was once considered to be an emotional disorder caused by improper attachment experiences to caregivers ${ }^{[4]}$. However, viral infections in utero, maternal valproic acid use and thalidomide use have all been implicated as independent cases of Autism Spectrum Disorder ${ }^{[2]}$. One study has commented that environmental factors encouraged other researchers to postulate that non-genetic mechanisms may produce an autistic syndrome ${ }^{[5]}$. However, the literature review did not yield articles that dealt with the connection between childhood neglect and the development of autism spectrum discord 


\section{Case Presentation}

The patient is a 5-year-old Caucasian male weighing 62.4 pounds, 42 inches tall and is currently enrolled in kindergarten. Approximately 3-5 months before his initial encounter with the pediatrician, he suffered from apraxia and was not toilet trained. He first presented to his pediatrician in June 2016 for a second opinion on Reactive Attachment Disorder and sensory processing issues. He currently has sensory processing problems that led to developmental delays and dyspraxia. His physical examination was unremarkable.

His medical history was positive for constipation, pica, Post Traumatic Stress Disorder, Reactive Attention Deficit Hyperactivity Disorder, Autism Spectrum Disorder, Shaken Baby Syndrome, Reactive Attachment Disorder and Sensory Processing Disorder. His pediatrician diagnosed him with Autism Spectrum Disorder while a psychologist made the other psychiatric diagnoses. Family history included bipolar disorder, antisocial personality disorder, Attention Deficit Hyperactivity Disorder, Conduct Disorder, substance abuse and non-specific learning disabilities on both sides of the family. The patient was prescribed Polyethylene Glycol 3350 Powder as needed for his constipation. He has never had any surgeries and has no known drug allergies.

The patient's mother was put on bed rest at 23 weeks of gestation after going into preterm labor on multiple occasions. She used MDMA and alcohol in the first trimester of her pregnancy but stopped once pregnancy was confirmed. He was born at term weighing 9 pounds and 15 ounces and met all motor developmental milestones, but language milestones were delayed. He spent time between his mother and father's home for his first three and a half years since his parents were separated. He also has an older half-brother who lived in the home at the same time.

He was consistently subjected to chronic neglect and physical abuse where he was locked into bedrooms unattended by an adult or was left with strangers not involved in his care. He was subjected to spending months on a feces covered mattress. He suffered from malnourishment, physical abuse and was able to escape the residence and was found on the street unattended. The patient was referred to interventional services for his developmental delay, while in the care of his mother, but attendance was sparse. He was also absent for almost half the time that he was enrolled at half-day kindergarten. He spent a short time in foster care after the Department of Child and Family Services got involved in his care. His paternal grandmother was subsequently awarded custody of him. He currently resides with his grandmother, grandfather and 22-year-old aunt and has a good relationship with all of them. There is no current relationship with his father and his mother has since been incarcerated. He was transferred to a learning center where he was placed in a half-day blended pre-kindergarten class where special education services were deemed necessary due to his diagnosis of Reactive Attachment Disorder. He underwent a battery of cognitive and developmental testing, the results of which are indicated below.

\section{Cognitive Testing}

Wechsler Preschool and Primary Scale of Intelligence-4th edition the mean score for these tests is 10 with an average of 8-12. It was further divided into subtests, the scores of which are stated in parentheses below:
1. Fluid Reasoning - Picture Concepts (8) and Zoo Locations (9)

2. Visual Spatial - Object Assembly (15) and Cancellation (4)

\section{The Bracken Basic Concept Scale-3rd edition}

This scale was used to assess receptive and expressive language. The School Readiness Composite subtest was divided into Receptive Language, which he scored in the 61st percentile, and Expressive Language, which was in the 73rd percentile. The Total Composite denoted higher-level concepts where his Receptive Language score in the 45th percentile was significantly higher than the Expressive Language subtest in the 14th percentile.

\section{Language Testing}

\section{The Comprehensive Assessment of Spoken Language}

He scored in the 19th percentile for the Pragmatic Judgment subtest.

\section{Subtests of the Social Perception and Attention/Executive Function Scales of the NEPSY-II}

The results are denoted with scores in parentheses:

1. Affect cognition (12) - 75th percentile

2. Theory of Mind (6) - 9th percentile

3. Statue (5) - 5th percentile

4. Body Part Naming (3) - Below first percentile

5. Body Part Identification (6) - 9th percentile

\section{Visual Motor Function Testing}

Wide Range Assessment of Visual Motor Abilities

He scored in the 30th percentile for the Visual-Spatial subtest, 10th percentile for the Visual-Motor subtest and the 13th percentile for the Fine-Motor subtest.

\section{Parent Report Measures}

The patient's grandmother participated in the Schedule for Affective Disorders and Schizophrenia for School-Aged Children (K-SADS), the Behavioral Assessment System for Children-3 (BASC-3) and Gilliam Autism Rating Scale-3 (GARS-3) to assess his emotional, behavioral and social functioning at home. The BASC-3 and GARS-3 Anxiety subscales were markedly elevated. The Maladaptive Speech was rated as problematic and the Restricted/Repetitive Behaviors subscale was also elevated. The patient was seen to have difficulty adjusting to changes in routine and lined up his toys excessively.

Furthermore, the patient was reported by his grandmother to worry about his parents, required excessive reassurance, has nightmares, demonstrates poor eye contact, is distracted and has problems with attention, hyperactivity and impulsivity. She also commented that he sometimes makes negative statements about himself and practiced one episode of selfharm after he had phone contact with his half-brother.

\section{Teacher Report Measures}

His kindergarten teacher also completed the BASC-3, GARS-3 and Child Adolescent Symptom Inventory-5 (CASI-5) to assess emotional and behavioral functioning at school. His attention, hyperactivity and impulsivity were rated to be milder at school. The Social Communication subscale of GARS-3 was elevated with difficulties in understanding humor and literal language. BASC-3 was normal. 


\section{Discussion}

The results of cognitive and developmental testing of a 5year-old Caucasian male with Autism Spectrum Disorder show low percentiles in Expressive Language and Pragmatic Judgment subtests. Furthermore, he scored low on 4 out of the 5 subtests of the Social Perception scales. Maladaptive Speech and Restricted/Repetitive Behavior subscales were seen to be elevated. All of these elements describe the three main hallmarks of Autism Spectrum Disorder. In this particular case report, several questions have been raised. One such question is, can a pediatrician make a reliable diagnosis of Autism Spectrum Disorder or should a licensed clinical child psychiatrist be responsible for such a task? Pediatricians are usually the first medical care providers that a parent and child encounters. Parents and caregivers are usually able to recognize changes in development, language and cognition immediately. As a result, this should prompt the physician to probe deeper into the child's developmental status. One study recommended that developmental surveillance be done at each well child visit ${ }^{[6]}$. This should include both social and emotional development in addition to other parameters. Further testing such as lead screens, phenylketonuria testing and chromosomal analysis have also been recommended to rule out etiologies and therefore direct treatment and intervention ${ }^{[6]}$. One study further concluded that pediatricians could make the Autism Spectrum Disorder diagnosis with proper training, experience and additional diagnostic tools such as the DSM$\mathrm{V}$ [6]. With this particular patient, his well-child evaluation included developmental assessment with both social and emotional assessments in addition to nutrition, family checks and pediatric physical examination. His pediatrician has been in practice for over 20 years and made the diagnosis by consulting the DSM-V criteria and making deductions based on his school-mandated cognitive, developmental testing and caregiver reporting. Genetic linkages to Autism Spectrum Disorder have been established but little research has been done to deduce the role of nurture in the disorder. However, neurodevelopmental research states that child neglect can lead to deregulated neural systems during crucial development of the brain, which can lead to cognitive and behavioral deficits ${ }^{[7]}$. One study used animal models to determine the effect of early neglect on the brain and development of mental disorders. ${ }^{3}$ The study has determined that changes in the white matter of the brain have been seen in child abuse and neglect. They determined that the medial prefrontal cortex contains militated axons, which are necessary for normal prefrontal cortex function. Early neglect in these animal models has been shown to cause down-regulation of transcription factors involved in myelination oligodendrocyte production. Furthermore, their study indicated that many mental disorders have been linked to abnormal myelin. Many of these militated axons project to the prefrontal cortex and there have been studies that have shown differences in the prefrontal cortex of those afflicted with Autism Spectrum Disorder, Obsessive Compulsive Disorder and Attention Deficit Hyperactivity Disorder. $^{3}$ Finally, another study stated two theories involving autism and the role of abnormal myelination. ${ }^{3}$ The first theory stated that impaired conduction velocity in excitatory fibers can alter the firing of synapses in the brain leading to impaired regulation of GABA in particular and thereby other neurotransmitters can be affected as a result
[3]. That study also found abnormalities in the regulation of GABA ${ }^{[3]}$. The second theory states that myelin may form an inhibitory environment, which is related to axon sprouting. Axon sprouting is needed to form accurate synapses in the brain.

In addition, the patient was diagnosed with comorbid Reactive Attachment Disorder. This raises the question; can Reactive Attachment Disorder be a manifestation of Autism Spectrum Disorder or is it a completely separate entity? According to one study one of the requirements for a diagnosis of Reactive Attachment Disorder is "social neglect that is absence of adequate caregiving during childhood ${ }^{[8]}$. That study also stated that the three classification systems of DSM-V, DC:0-3 and ICD-10 suggests that Reactive Attachment Disorder should not be diagnosed in patients with Autism Spectrum Disorder because of a fear of mistaking Autism Spectrum Disorder for Reactive Attachment Disorder ${ }^{[8]}$. Their study found however, that specific symptoms such as restricted/repetitive movements, stereotypical behavior for autism, delayed speech milestones and unusual fears were found only in their Autism Spectrum Disorder group and not in the Reactive Attachment Disorder group. They concluded that both disorders could co-exist. As far as this case report is concerned, the child abuse and neglect can be an etiological factor for the Reactive Attachment Disorder and also may be a possible contributing factor in addition to established neurodevelopmental etiologies for autism spectrum disorder.

This particular case is unique in that there have been very few studies determining a link between autism spectrum disorder and child neglect. The limitations of this study include a single research subject instead of meta-analytical studies examining the specific link between child neglect and Autism Spectrum Disorder. In addition, more studies are needed examining the possibility of pseudo-autistic features versus Autism Spectrum Disorder in children who have suffered neglect. Another limitation is the use of animal models to determine the link between neglect impacting proteomic production leading to Autism Spectrum Disorder. It would be unethical to use children in this manner since most studies are invasive or must be conducted post-mortem. Finally, some of the behavioral information and details of the extent of child neglect and abuse provided on the patient in this case report is subjective to his current caretaker and may not be complete. Finally, one study has also stated that resilience in children who have suffered from child neglect can be instilled by a proper school environment and a supportive emotional environment with a reliable caregiver, therefore further studies into this matter can direct evidence-based treatment options ${ }^{[7]}$.

\section{Conclusion}

Autism Spectrum Disorder is multifactorial in nature. There are highly correlated neurobiological etiologies but not much information is provided for the role of nurture in Autism Spectrum Disorder. By deducing the specific etiologies and risk factors of Autism Spectrum Disorder, treatment such as behavioral interventions, psychotherapy or cognitive development can be formulated based on evidence-based recommendations and better assist patients in assimilating into society. 


\section{Acknowledgements}

The author would like to thank Dr. Todd Ochs for his support and mentorship.

\section{References}

1. Diagnostic and statistical manual of mental disorders. American Psychiatric Association. 5th edition. Arlington, VA: American Psychiatric Publishing, 2013.

2. DiCicco-Bloom E, Lord C, Zwaigenbaum L et al. The Developmental Neurobiology of Autism Spectrum Disorder. The Journal of Neuroscience 2006; 26(26):6897-6906.

3. Bordner KA, George ED, Carlyle BC et al. Functional Genomic and Proteomic Analysis Reveals Disruption of Myelin-Related Genes and Translation in a Mouse Model of Early Life Neglect. Frontiers in Psychiatry. 2011; 2(18):1-18.

4. Bettelheim B. The empty fortress: infantile autism and the birth of the self. Oxford: Free Press of Glencoe, 1967.

5. London E, Etzel RA. The Environment as an Etiologic Factor in Autism: A New Direction for Research. Environmental Health Perspectives. 2000; 108(3):401404.

6. Sandler AD, Brazdziunas D, Cooley WC et al. The Pediatrician's Role in the Diagnosis and Management of Autistic Spectrum Disorder in Children. Pediatrics. 2001; 107(5):1221-1226.

7. Spratt EG, Friedenberg SL, Swenson CC et al. The Effects of Early Neglect on Cognitive, Language, and Behavioral Functioning in Childhood. Psychology (Irvine). 2012; 3(2):175-182.

8. Mayes SD, Calhoun SL, Waschbusch DA et al. Autism and reactive attachment/disinhibited social engagement disorders: Co-occurrence and differentiation. Clinical Child Psychology and Psychiatry. 2016; 22(4):620-631. 